\title{
Issues to Consider in Designing Immunotherapy Clinical Trials for Glioblastoma Management
}

\author{
Adela $\mathrm{Wu}^{1 *}$, Michael Lim ${ }^{2}$ \\ ${ }^{1}$ Johns Hopkins University School of Medicine, Baltimore, USA \\ ${ }^{2}$ Department of Neurosurgery, Johns Hopkins Hospital, Johns Hopkins University School of Medicine, \\ Baltimore, USA \\ Email: "awu30@jhmi.edu, mlim3@jhmi.edu
}

Received 27 April 2016; accepted 7 August 2016; published 10 August 2016

Copyright (C) 2016 by authors and Scientific Research Publishing Inc.

This work is licensed under the Creative Commons Attribution International License (CC BY). http://creativecommons.org/licenses/by/4.0/

\section{(c) (i) Open Access}

\begin{abstract}
Glioblastoma (GBM) is the most common primary brain malignancy in adults and has a poor prognosis despite standard of care treatment. The mainstay of GBM treatment has relied on maximum surgical resection and chemotherapy and radiation. Cancer immunotherapy has made great strides since the advent of anti-PD-1, anti-CTLA-4, and other immune checkpoint inhibitors. With the advancement of novel therapeutics, more clinical trials for patients have opened as well. An important future direction of clinical trials is the ability to identify appropriate patients to optimize treatment response and minimize toxicities. This review describes considerations in designing future GBM clinical trials in not only immunotherapy but also with other promising treatments. We will discuss factors, such as pseudoprogression, genetic and circulating biomarkers, and the commensal microbiome of patients in the setting of clinical trial design.
\end{abstract}

\section{Keywords}

Cancer, Glioblastoma Management, Treatment Response, Prognosis, Pseudoprogression, Biomarker, Microbiome, Immunotherapy, Chemotherapy

\section{Introduction}

Glioblastoma (GBM) is the most common primary brain malignancy (80\%) with a high mortality of 12 - 15 months median survival [1] [2]. According to the National Cancer Institute, 2 - 3 people per 100,000 are diagnosed each year with GBM. The majority of GBM cases arise sporadically, from a combination of a multitude and a variety of mutations, such as in p53, vascular endothelial growth factor (VEGF), and EGFR [2]. Current

\footnotetext{
*Corresponding author.
}

How to cite this paper: Wu, A. and Lim, M. (2016) Issues to Consider in Designing Immunotherapy Clinical Trials for Glioblastoma Management. Journal of Cancer Therapy, 7, 573-585. http://dx.doi.org/10.4236/jct.2016.78060 
standard of care includes surgical resection, radiation, and chemotherapy with temozolomide [3].

However, GBM is known for being recurrent and treatment-resistant, due to the infiltrative nature and heterogeneity of tumor composition and numerous subpopulations of cancer stem cells (CSCs) as well as the pervasive state of immunosuppression and T cell exhaustion in cancers [4] [5] The pathogenesis itself of GBM critically involves the growth and proliferation of a special subset of these pluripotent CSCs, which act as tumorinitiating cells and also promote angiogenesis for the tumor [2]. In addition, the GBM tumor microenvironment encompasses a variety of immunosuppressive characteristics. Increased numbers of immunosuppressive cytokines, such as IL-6 and IL-10, as well as increased ratios of regulatory T cells as opposed to effector T cells are examples of ways GBM microenvironment silences the body's immune response [6]. In recent years, immune checkpoint blockade as oncologic therapy has rapidly progressed in order to address this particular resistance to standard GBM care. Examples of critical immune checkpoint inhibitor drugs include anti-Programmed Death 1 (anti-PD-1) and anti-cytotoxic T lymphocyte antigen 4 (anti-CTLA-4) with many more drugs in the pipeline [7].

As the repertoire of GBM therapeutics continues to grow, ways of monitoring prognosis and especially treatment response should follow as well. Immunotherapy in particular can be modulated with a variety of circulating factors and molecules, such as a recently studied cholesterol esterification enzyme and even intestinal microorganisms [8] [9]. Monitoring GBM treatment response over time is still a much-needed topic of research in GBM and cancer management in order to draw conclusions about the clinical trials' endpoint, patients' survival outcomes. Anti-angiogenic treatment in GBM has been followed with proteomic analysis of specific proteins that were involved in well-known pathways of metabolism, apoptosis, and angiogenesis [10] Other monitoring methods include utilizing magnetic resonance imaging (MRI) and fluorescence molecular imaging [11] [12]. However, describing true disease progression is sometimes uncertain with monitoring treatment response through imaging due to phenomena such as pseudoprogression in MRI. In addition, in order to arrive at meaningful endpoints, clinical trials should take into consideration methods to follow patient prognosis and treatment response, such as through alterations in genetic expression or through cellular biomarkers like ctDNA. In the context of individualized patient parameters, treatment response to cancer immunotherapy being manipulated by the makeup of the intestinal microbiota is another fruitful area to be explored further. This review therefore strives to highlight different important issues, such as radiological and biological aspects of patient selection, prognosis, and treatment response efficacy, to consider in designing clinical trials for GBM immunotherapy and cancer treatment.

\section{Discussion}

\subsection{Patient Selection}

According to ClinicalTrials.gov, more than 200,000 clinical trials are currently running in 193 countries, with 80\% of studies involving drug, surgical, behavioral, or instrumental intervention in a disease process [13]. A significant aspect of designing these clinical trials is oriented on potentially prognostic subject and patient characteristics on treatment response to intervention [14].

In addition to traditional factors such as age, performance status, and amount of tumor resected, aspects such as MGMT status, IDH status, and lymphopenia may play important roles in future clinical trials.

In GBM management, it is well known that methylation of O6-methylguanine-DNA methyltransferase (MGMT) promoter predicts better response to temozolomide chemotherapy as well as reflects fairer prognosis [15]-[18]. Specific tumor makeup can also inform the types of medication administered during a clinical trial. In a group of 10 patients who had already undergone surgery and chemoradiation, the decision to add the immunotherapeutics, bevacizumab and erlotinib, was based on the expression of epidermal growth-factor (EGFRvIII) and methylated MGMT promoter in GBM tumor samples [19]. Since these studies involving methylated MGMT promoter and GBM management demonstrated survival ranging up to 18 - 21 months, necessitating clinical trials that will incorporate sufficiently long periods of patient follow-up [20] [21].

However, not all GBM tumors contain methylated MGMT promoters, ranging from $28.7 \%$ to $50.6 \%$ of tumors, so basing clinical trials solely on this one marker would be shortsighted [22] [23]. Some investigators even urge practitioners to rethink the standard of ubiquitously prescribing temozolomide to all GBM patients in light of studies that demonstrate no added benefit for certain patient populations, such as the elderly with unmethylated MGMT promoters [24].

It would also be fruitful to examine patient selection based on somatic mutations of isocitrate dehydrogenase 
(IDH), which are commonly seen in glial tumors. According to a clinical trial that compared chemoradiotherapy and radiation in 291 patients, $74 \%$ of the patients had a positive IDH-mutation status, which correlated to a longer overall survival. Median survival was not affected by chemoradiotherapy in those without the IDH mutation [98].

Another interesting parameter that arises in oncological clinical trials is treatment-related lymphopenia (TRL), reflected by lymphocyte counts in patients undergoing chemotherapy and/or radiation. For example, in a study on patients with pancreatic adenocarcinoma, a lower total lymphocyte count after adjuvant chemo-radiation was associated with a lower median survival (14 vs 20 months, $\mathrm{p}=0.48$ ) [99]. Likewise, for malignant glioma, non-small cell lung cancer, as well as unresectable pancreatic cancers, patients with low TRL demonstrated shorter periods of survival from their diseases [100]. Specifically, the number of CD4+ T cells decreased while the pool of CD8+ T cells stayed stable when TRL was examined in glioma clinical trials [101]. Especially in future clinical trials that could involve immunotherapy, incorporating lymphopenia as a marker of immunologic change resulting from effective treatment would be an important factor to consider.

\subsection{Monitoring Disease Progression during Clinical Trials}

It is paramount to monitor disease progression throughout a trial before considering the overall impact of a particular therapy on patient survival outcomes. However, GBM clinical studies, especially those that include immunotherapeutics as intervention, lack reliable methods of immunologic assessment [25] Therefore, the longterm follow-up protocol for these GBM clinical trials commonly involve magnetic resonance imaging (MRI) to monitor disease status in addition to clinical and functional assessment [26] [27].

\subsection{Pseudoprogression}

Pseudoprogression (PsP) is a radiological phenomenon of the apparent increase in size of contrast-enhancing tumor on imaging due to treatment and not tumor growth, reported for the first time in 1979 [28] [29]. There are no accompanying and associated neurological symptoms in cases of pseudoprogression. Vasogenic edema in the glioma tumor bed and abnormal permeability of surrounding vasculature contributes to the appearance of pseudoprogression [28]. Radiation therapy causes endothelial injury in vessels as well as vasodilation and inflammatory disruption of the blood-brain barrier [30]. Some traditionally-used brain soft tissue imaging could muddle the true underlying mechanism of contrast enhancement that appears radiologically. Pseudoprogression is associated with higher rates of survival in glioma patients and could actually reflect radiochemotherapy treatment efficacy [31] [32]. Therefore, examining the mechanism and the usefulness of different imaging modalities in future clinical trials for GBM and immunotherapy is critical.

There are guidelines to describe response criteria of gliomas to treatment. These response criteria have been frequently referred to in landmark clinical trials for glioma management. The Macdonald criteria, published in 1990, used the enhancing tumor area, radiologically determined through enhanced CT or MRI scan, as the primary basis of tumor treatment response [33]. A significant increase of at least $25 \%$ in the contrast-enhancing lesion area was proposed as a marker of tumor progression. The major drawback of relying solely on Macdonald criteria and its most significant factor of contrast-enhancing tumor area in determining disease progression and subsequent management is the nonspecificity of the radiological contrast enhancement. Contrast enhancement on imaging could also be seen whenever contrast permeates a disrupted blood-brain barrier, which commonly occurs after the radiation, chemotherapy and immunotherapy utilized in GBM management. More limitations of the Macdonald criteria have also arisen concerning inter-observer variability and no assessment guidelines for multifocal tumors, non-enhancing portions of tumor, or tumors with irregular borders [34]. The Response Assessment in Neuro-oncology (RANO) Working Group proposed updated standardized response criteria for brain tumor clinical trials in 2012 [34]. The RANO recommendations include imaging after surgery within 72 hours to determine the extent of resection for contrast-enhancing tumors, diffusion weighted imaging to characterize non-enhancing tumor components and areas of ischemia, and evaluations of disease progression and volumetric-based assessments of residual tumor burden.

Following standard therapy of radiochemotherapy for GBM management, 20-40\% of those patients had increased enhancement on the first post-treatment follow-up MRI scans, of which half were due to the phenomenon of pseudoprogression [102]. Immunotherapy used to treat GBM, such as the administration of geneticallyengineered $\mathrm{T}$ cells in mice, resulted in T1 gadolinium enhancement and T2 hyperintensity 24 hours following treatment, which resolved 2 - 3 days afterward [35]. In fact, Administration of proinflammatory cytokines 
increased contrast enhancement that lasted up to 3 months post-treatment [34]. Two patients underwent surgery, radiation, chemotherapy as well as IL-4 toxin immunotherapy within the tumor bed, and their follow-up MRI scans also showed increased enhancement. However, to determine whether the enhancement was due to true tumor progression or pseudoprogression, the investigators used magnetic resonance spectroscopic imaging (MRSI) to test the metabolic activity of the delineated tissue, concluding that MRSI could be a useful tool in differentiating pseudoprogression following intralesional immunotherapy [103]. Certainly as new immunotherapeutics are being tested in clinical trials, it would be paramount to anticipate and closely assess pseudoprogression. It may be even more likely for pseudoprogression, which is mediated by inflammatory processes, to appear more often post-immunotherapy, necessitating thoughtful choices for follow-up imaging modalities [104].

Post-gadolinium MRI cannot effectively differentiate between treatment-associated pseudoprogression or early tumor and disease progression following radiation, chemotherapy or immunotherapy [36] [37]. Several new and alternative imaging methods to determine the incidence of true PsP have been proposed and include dynamic MRI as well as modified PET imaging with attention paid to how best to characterize the vascular changes occurring in the brain and tumor tissue due to chemotherapy and radiation that could result in PsP (Table 1). Dynamic contrast-enhanced T1 MRI resulted in lower volumetric plasma volume (Vp) perfusion values as well as lower plasma volume and time-dependent leakage constant values (Ktrans) associated with PsP [30]. Dynamic susceptibility-weighted contrast-enhanced MRI with ferumoxytol molecules was used in a study of 56 GBM patients who had received chemotherapy and radiation. Relative cerebral blood volume (rCBV) values were collected, with 1.75 rCBV assigned as the cutoff value for determining PsP [32].

A common observation across groups revolves the higher incidence of pseudoprogression noted in patients with methylation of MGMT-promoter within glioma [37]. One group found that $31 \%$ of patients with methylated GBM demonstrated pseudoprogression in contrast to just 5\% incidence in patients with non-methylated tumors [29]. In fact, there is as high as a $91.3 \%$ probability of pseudoprogression in tumors with methylatedMGMT glioma expression compared to a 59\% probability of early tumor progression in those with unmethylated MGMT promoter [38]. Pseudoprogression itself appeared to be correlated and more common amongst patients who had methylated MGMT promoter ( $91 \%$ vs. $41 \%$ unmethylated), possibly indicating the efficacy of the chemoradiotherapy on decreasing glioma tumor burden [39]. This difference in pseudoprogression rates based on methylation of the MGMT promoter could be correlated to treatment efficacy on diminishing tumor burden, thereby establishing MGMT-promoter methylation as a key factor in designing directed clinical trials based on tumor composition and also other biomarkers.

\subsection{Predicting Treatment Response and Clinical Trial Endpoints of Survival}

The gold standard of measuring treatment efficacy in clinical trials is overall survival (OS). OS as a data point is objective, but obstacles to utilizing OS as a measure include its need for large randomized trials with long follow-up periods [102].

In cancer trials, OS and PFS may necessitate follow-up periods that are too prolonged to be clinically feasible. Alternatives could be examining survival data at a predetermined time point, such as six months following

Table 1. Shows cited references for each category of alternative methods of imaging and delineating disease progression and pseudoprogression.

\begin{tabular}{|c|c|}
\hline \multicolumn{2}{|c|}{ Alternatives to post-gadolinium MRI to differentiate true progression from PsP } \\
\hline Methods & Numbered References (refer to bibliography) \\
\hline Dynamic O-(2-[18F]fluoroethyl)-L-Tyrosine PET & [29] [90] [97] \\
\hline $\begin{array}{c}\text { Dynamic contrast enhanced T1 magnetic resonance imaging (DCE MRI) } \\
\text { (enhanced with i.e. ferumoxytol) }\end{array}$ & [30] [32] [89] [93] [95] \\
\hline Cut-off values of apparent diffusion coefficients (ADCs) & $\begin{array}{c}\left.\text { [88] (ADC mean value } \leq 1300 \times 10^{-6} \mathrm{~mm}^{2} / \mathrm{s}\right) \\
{[96]\left(\mathrm{ADC}_{1000} \leq 929 \times 10^{-6} \mathrm{~mm}^{2} / \mathrm{sec},\right.} \\
\left.\text { ADC }_{3000} \leq 645 \times 10^{-6} \mathrm{~mm}^{2} / \mathrm{sec}\right) \\
904] \text { (ADC 5th percentile value } 892 \times 10^{-6} \mathrm{~mm}^{2} / \mathrm{sec} \text {, } \\
907 \times 10^{-6} \mathrm{~mm}^{2} / \mathrm{sec} \text { ) }\end{array}$ \\
\hline Multi-parameter aggregation and analysis & [91]-[93] \\
\hline
\end{tabular}


intervention. The primary endpoint of Phase II clinical trials in GBM management is statistically significant progression-free survival at 6 months (PFS-6) [40]-[42]. Secondary endpoints could include pharmacokinetics, quantifications of tumor response to therapeutics [40] [41]. PFS, necessarily based on imaging or practitioners' clinical assessment, such as by MRI, leads to earlier, truncated results than OS used as primary endpoints, arguably becoming a less clinically useful endpoint. One group has proposed an alternative to PFS and OS by combining the two parameters into a separate clinical endpoint that will retain the quick data turn-around and ease of monitoring disease progression that PFS offers with the clinical significance of OS [43]. Particularly with clinical trials that involve immunotherapy, there are a number of long-term survivors with survival trends that exceed historical data from trials involving radiochemotherapy [105]. Hence, in evaluating clinical data about survivorship, it would be more useful to use the area-under-curve as the parameter.

This review then elaborates on several biological and genetic markers that could be harnessed in order to derive meaningful data on various endpoints and patient outcomes from clinical trials in GBM and cancer management with immunotherapy and other regimens.

\subsection{Alterations in Genetic Expression as Markers of GBM Prognosis}

Well-studied markers of cancer prognosis include various alterations in genetic expression, such as the methylated MGMT promoter sequence, which is predictive of better patient outcome in therapy. Mutations of isocitrate dehydrogenase (IDH1 and IDH2), which can be seen in over 90\% of GBM tumor samples, predicted better overall survival as well as progression-free survival for GBM patients according to a recent meta-analysis [44]-[46]. However, the association between IDH mutations and long-term survival is not for certain and statistically insignificant for some patients; $5.9 \%$ of long-term survivors ( $>3$ years survival) had IDH mutations whereas $1.16 \%$ of the non-long-term survivors had the mutations $(p=0.144)$ [47]. Mutations in the TERT promoter, which is the most common GBM mutation, in conjunction with IDH mutation and EGFR amplification, correlated with worse prognosis and shorter survival times [48]. However, another study shows the opposite, in which TERT promoter mutation and EGFR amplification indicated better outcome [49]

Mutations in the APOBEC family of antiviral DNA cytosine deaminases have become a popularly researched biomarker of tumor burden and prognosis of late as well. APOBEC3G specifically has been found to be greatly elevated in T-cells within high-grade serous ovarian neoplasms and also were associated with better patient outcomes [50]. The underlying hypothesis to the correlation was that T-cell infiltration is favorable for diminishing tumor burden. However, elevated levels of APOBEC3B are present and indicative of poor prognosis in many cancers, such as breast or ovarian cancers [51]. Interestingly, those who carry deletions of APOBEC3B had hypermutation of tumors and could have better response to immunotherapies due to immune system activation [51]. Unfortunately, there are no studies of APOBEC's role as biomarkers in GBM to date yet. Different types of mutations could also affect immune response and GBM treatment efficacy. In a group of patients with metastatic gastrointestinal cancers, it was found that 9 out of 10 patients had immunogenic epitopes that were completely unique to each patient and to which the patient's CD4+ and CD8+ T cells could specifically target, potentially paving the way for personalized therapeutics in the future [106]. A separate study examined the somatic mutational load, which was determined following whole-exome sequencing, in tumors from patients who had long-term clinical benefit as well as patients who had lesser clinical benefit from immunotherapy [107]. The somatic mutations give rise to neoantigens, of which a specific set conferred greater efficacy of CTLA-4 blockade. Thus, utilizing sequencing tools and genetic analysis of tumor samples to determine the specific types of mutations could translate to differing treatment efficacy in GBM management. Furthermore, understanding the types of mutations is an aspect to be considered for GBM clinical trials.

\subsection{Role of Circulating Markers}

As the field of serum proteomics advances, other strong emerging candidates for biomarkers of malignancy include circulating tumor cells (CTCs) and cell-free circulating tumor DNA (ctDNA). ctDNA is released into the bloodstream after primary and metastatic tumors undergo apoptosis or necrosis, and CTCs become part of the circulation following the growth and movement of potential metastatic cancer cells. Numerous cancers, including gastric cancer, prostate cancer, breast cancer, and esophageal cancer, demonstrate elevated ctDNA in patients' serum [52]-[55]. It is possible to also use ctDNA as a method to follow the progression over time of some malignancies, such as non-small cell lung cancer (NSLC), for the composition of ctDNA isolated from patients' 
Table 2. Cited references for separate roles circulating tumor DNA (ctDNA) can play in monitoring different types of non-GBM cancers.

\begin{tabular}{cc}
\hline Role of ctDNA & Examples of cancer types (reference number) \\
\hline Monitor disease progression & Non-small-cell lung cancer [56] \\
Melanoma [58] \\
Correlate with tumor burden & Metastatic colorectal cancer [59] \\
Reflect prognosis and survival outcome & Metastatic breast cancer [57] \\
& Metastatic melanoma [61] \\
\hline
\end{tabular}

serum contained mutations in EGFR, KRAS, PIK3CA, and TP53, which are also found in the DNA of primary tumor tissue, with 76\% concordance [56] (Table 2). Dawson et al. also showed that ctDNA has enormous promise as a biomarker to monitor metastatic breast cancer. Not only did $97 \%$ of patients with somatic genetic mutations in their neoplasms also have detectable ctDNA, the ctDNA correlated more closely to actual tumor burden than even older, well-studied cancer markers, such as CA 15-3 [57]. Some groups have then shown that the levels of ctDNA correspond to disease progression, measured by clinical symptoms as well as radiographic evidence. The correlation between the serum biomarker ctDNA and progression is present in cancer patients who have undergone chemotherapy or immune checkpoint blockade therapy [58] [59]. For some patients, an increase in ctDNA can demonstrate disease progression up to 2-9 months earlier than the first evidence by imaging [57] ctDNA and CTCs can both also be associated with prognosis and likelihood of worse outcomes [60] [61]. In some cancers, namely metastatic uveal melanoma, ctDNA appears to be a better prognostic marker than levels of CTCs for quantifying prognosis [61]. However, the positive association between level of ctDNA and poor prognosis is not completely certain. A study in breast cancer patients with TP53 mutations in their tumors did not demonstrate a clear correlation between ctDNA and outcome [62].

\subsection{Measuring Prognosis of GBM with Cellular Biomarkers}

Glioblastoma (GBM) in particular has also received a great degree of investigation in terms of associating biomarkers with different measures of disease burden and prognosis. ctDNA appears to be useful in following the mutational patterns of glioblastoma and gliomas as well [63]. In another study, further study of GBM patients' plasma yield microRNAs that were significant in prognosis and quantifying tumor burden. 24 microRNAs were significantly linked to survival, while 11 microRNAs were diagnostic and expressed in GBM patients' serum [64]. In addition, high levels of FGL2 mRNA led to evidence of lower overall survival, presumably through promoting immunosuppression in the GBM tumor microenvironment [65]. Elevation of circulating endothelial cells (CECs), which are markers of endothelial damage, has been associated with poor prognosis in NSCLC, while in other cancers, the correlation was converse [66]. In GBM, apparently CEC levels elevated when there was poor response to treatment by a pan-VEGF receptor tyrosine kinase inhibitor [67]. Overexpression of ribosomal proteins, RPS11 and RPS20, also correlates with poor prognosis of GBM patients [68]. The number and ratio of $\mathrm{T}$ cells have also been another area of interest. While the ratio of $\mathrm{CD} 4+\mathrm{T}$ cells to regulatory $\mathrm{T}$ cells was low in recurrent GBM, when the same ratio-as well as CD8+/regulatory T cell ratio-was elevated, it correlated directly to improved survival measures [69]. However, apparently, the number of regulatory T cells on their own did not show an association with survival in primary GBM [70].

\subsection{Biomarkers of Treatment Response in GBM and Other Cancers}

Even so, another area that benefits from identification and investigation of suitable and reliable biomarkers would be correlation with treatment response and efficacy. Dawson et al. demonstrated a remarkable quality of ctDNA in metastatic breast cancer; the level of ctDNA was the first marker to show treatment response in 53\% of study participants [57]. A group following patients with metastatic melanoma found that lower ctDNA levels indicated better response to treatment and progression-free survival (PFS) [71]. Of note, in the aforementioned study, patients who received MAPK inhibitors demonstrated decreased ctDNA levels, whereas immunotherapy did not elicit the same treatment response [71]. Muddling the link between ctDNA and treatment response is the 
effect of chemotherapy on cancer cells. It is theorized that the amount of methylated ctDNA first increases in patients responding well to chemotherapy and then falls once the tumor burden shrinks in the chemosensitive patients [72].

There are a variety of other biomarkers potentially reflecting treatment response in a variety of cancers [73]-[76]. In GBM specifically, a group found that phosphorylation of two transcription factors, STAT-1 and STAT-5, can delineate response to immunotherapy and dendritic cell vaccines [77]. Survival past two years was seen in patients who demonstrated a greater pSTAT-5 ratio but a lower pSTAT-1 ratio. Plasma exosomal levels and mRNA expression are other biomarkers to measure GBM treatment response to a dendritic cell-based vaccine. Exosomal protein and mRNA component fractions, such as those of IL-8 and TGF-beta, positively reflected immunologic response to the vaccine [78]. miRNA can also predict response to temozolomide in treating GBM [79] Returning to the discussion on alterations in genetic expression, it appears that EGFR amplification and overexpression led to better response to temozolomide [80]. Finding biomarkers to monitor the effectiveness of immunotherapy on GBM disease progression would be very useful, but, thus, far, it appears that only following antibody titers, where the titers increased with greater and longer duration of treatment, is fruitful. Studies examining whether cytokine levels rose in conjunction with administration of dendritic cell vaccines or personalized peptides did not yield statistically significant results [81].

Overall, as research techniques continue to advance, there is a multitude of well-established as well as more recently-studied circulating biomarkers and genetic alterations that could delineate trends in different cancer types. For GBM specifically, much attention has been given to MGMT promoter methylation as well as mutations, such as IDH1 and IDH2, in predicting prognosis as well as monitoring disease progression. Given the variety of different molecules and patient plasma components, including miRNA, ribosomal proteins, and circulating tumor DNA, there is still much more room for investigation with the hope of incorporating some of the more consistently reliable biomarkers in critical GBM clinical trials.

\subsection{Gut Microbiota and Cancer Therapy}

Another potential component to consider for future clinical trials involving GBM immune checkpoint therapy in particular would be the role and influence of gut microbiota, a truly individualized aspect in patient-centered onco-therapeutics. Colonization by specific intestinal microorganisms, such as Bacteroides fragilis, and their components, bacterial polysaccharide (PSA) leads to a guided development and maturation of the immune system, including the establishment of the host's Th1/Th2 cell balance [82]. On a more specific level, commensal bacteria, including Bacteroides thetaiotaomicron, can also direct targeted immune response to certain Grampositive bacteria by inducing intestinal endothelial cells (IECs) to release antimicrobial proteins [83].

Given the nature of the cancer tumor microenvironment and its suppression of the immune system, harnessing the immunomodulatory aspects of commensal gut microbiota appears to be the next big step in immuno-oncology investigation. First off, the administration of immune checkpoint therapeutics changes the composition of gut microbiota. When patients with advanced melanoma were given doses of ipilimumab, the CTLA-4 checkpoint inhibitor caused fluctuations in antibody levels to their enteric flora [84], resulting in adverse side effects, such as diarrhea and colitis that were different in process than inflammatory bowel disease. Vetizou et al then demonstrated that the gut microbiome was critical for the anti-carcinogenic effects of ipilimumab in murine models of sarcoma, melanoma as well as colon cancer [85]. In particular, the presence of $B$. fragilis, B. thetaiotaomicron, and Burkholderiales induced TH1 immune response in lymph nodes, enhanced maturation of antigen-presenting dendritic cells within tumors, and overall stimulated the immune response against tumor cells. Another specific organism, Bifidobacterium, was found to be associated with antitumor immunity in a murine melanoma model established in genetically similar animals from two different facilities (JAX and TAC). In fact, the oral administration of Bifidobacterium on its own had comparable antitumor effects as administration of anti-PD-L1 antibody immune checkpoint blockade [86]. Fecal transplants between the TAC and JAX mice, the guts of which contained the commensal bacteria participating in antitumor immunity, resulted in not only slower tumor growth but also increased degree of tumor-specific T cell infiltration and response as anti-PD-L1 blockade on its own.

With landmark studies and other investigations still ongoing, cancer immunotherapy can be enhanced greatly with attention paid to particular intestinal commensal microorganisms that can contribute to anti-tumor effects. Future clinical trials may become more patient-centric with more time and understanding of how the gut microbiome plays a critical role in the immune response of therapy. 


\section{Conclusion}

The research in and development of new and more effective GBM therapeutics is ever-evolving. GBM clinical trials should follow this advancement as well and incorporate different aspects from the patient selection to the derivation of primary endpoints in order to address the individualization of GBM patients and the unique nature of GBM neoplasms. In a variety of cancers, it has been shown that pseudoprogression on imaging and numerous biomarkers have the potential to allow clinicians and scientists to monitor oncology patients' prognosis and response to different types of therapy. Additionally, the intestinal microbiome appears to have exciting promise to modify and enhance the very efficacy of cancer therapeutics, including most notably immunotherapeutics, themselves. Consideration of all parameters together is a potent way to develop effective GBM and cancer treatments further. For example, Linhares et al. found that updated RANO criteria described pseudoprogression in more predictable terms in their study group and suggested that radiological, not only biological, biomarkers could be identified in the future to follow disease progression and treatment efficacy [87]. Many nuances are left to be examined in these prognostic and treatment-response aspects as applicable to different cancers, previously studied already or not. GBM management, in particular, has been under intense interest due to the disease's relentless and devastating nature, and future clinical trials could certainly benefit from studying these markersMGMT methylation, PsP on imaging, circulating and genetic mutation biomarkers, and species of microorganisms — of patient selection, clinical outcome, and treatment response.

\section{References}

[1] Malhotra, K., Shamkant, B.N., Chau, D.H., et al. (2016) Constraint Based Temporal Event Sequence Mining for Glioblastoma Survival Prediction. Journal of Biomedical Informatics, 61, 267-275. http://dx.doi.org/10.1016/j.jbi.2016.03.020

[2] Aliferis, C. and Trafalis, D.T. (2015) Glioblastoma Multiforme: Pathogenesis and Treatment. Pharmacology \& Therapeutics, 152, 63-82. http://dx.doi.org/10.1016/j.pharmthera.2015.05.005

[3] Stupp, R., Hegi, M.E., Gilbert, M.R. and Chakravarti, A. (2007) Chemoradiotherapy in Malignant Glioma: Standard of Care and Future Directions. Journal of Clinical Oncology, 25, 4127-4136. http://dx.doi.org/10.1200/JCO.2007.11.8554

[4] Fouse, S.D., Nakamura, J.L., James, C.D., et al. (2014) Response of Primary Glioblastoma Cells to Therapy Is Patient Specific and Independent of Cancer Stem Cell Phenotype. Neuro-Oncology, 16, 361-371. http://dx.doi.org/10.1093/neuonc/not223

[5] Wherry, E.J. and Kurachi, M. (2015) Molecular and Cellular Insights into T Cell Exhaustion. Nature Reviews Immunology, 15, 486-499. http://dx.doi.org/10.1038/nri3862

[6] Razavi, S.M., Lee, K.E., Jin, B.E., Aujla, P.S., Gholamin, S. and Li, G. (2016) Immune Evasion Strategies of Glioblastoma. Frontiers in Surgery, 3, 11. http://dx.doi.org/10.3389/fsurg.2016.00011

[7] Topalian, S.L., Drake, C.G. and Pardoll, D.M. (2015) Immune Checkpoint Blockade: A Common Denominator Approach to Cancer Therapy. Cancer Cell, 27, 450-461. http://dx.doi.org/10.1016/j.ccell.2015.03.001

[8] Yang, W., Bai, Y., Xiong, Y., et al. (2016) Potentiating the Antitumor Response of CD8+ T Cells by Modulating Cholesterol Metabolism. Nature, 531, 651-655. http://dx.doi.org/10.1038/nature17412

[9] Snyder, A., Pamer, E. and Wolchok, J. (2015) Could Microbial Therapy Boost Cancer Immunotherapy? Science, 350, 1031-1032. http://dx.doi.org/10.1126/science.aad7706

[10] Demeure, K., Fack, F., Duriez, E., et al. (2016) Targeted Proteomics to Assess the Response to Anti-Angiogenic Treatment in Human Glioblastoma (GBM). Molecular \& Cellular Proteomics, 15, 481-492. http://dx.doi.org/10.1074/mcp.M115.052423

[11] Gzell, C.E., Wheeler, H.R., McCloud, P., Kastelan, M. and Back, M. (2016) Small Increases in Enhancement on MRI May Predict Survival Post Radiotherapy in Patients with Glioblastoma. Journal of Neuro-Oncology, 128, 67-74.

[12] Li, Y., Du, Y., Liu, X., et al. (2015) Monitoring Tumor Targeting and Treatment Effects of IRDye 800CW and GX1Conjugated Polylactic Acid Nanoparticles Encapsulating Endostar on Glioma by Optical Molecular Imaging. Molecular Imaging, 14, 356-365.

[13] ClinicalTrials.gov, U.S. National Institutes of Health, Feb 2016.

[14] Boessen, R., Heerspink, H.J.L., De Zeeuw, D., et al. (2014) Improving Clinical Trial Efficiency by Biomarker-Guided Patient Selection. Trials, 15, 103. http://dx.doi.org/10.1186/1745-6215-15-103

[15] Barault, L., Amatu, A., Bleeker, F.E., et al. (2015) Digital PCR Quantification of MGMT Methylation Refines Prediction of Clinical Benefit from Alkylating Agents in Glioblastoma and Metastatic Colorectal Cancer. Annals of Oncology, 26, 1994-1999. http://dx.doi.org/10.1093/annonc/mdv272 
[16] Brigliadori, G., Foca, F., Dall’Agata, M., et al. (2016) Defining the Cutoff Value of MGMT Gene Promoter Methylation and Its Predictive Capacity in Glioblastoma. Journal of Neuro-Oncology, 128, 333-339. http://dx.doi.org/10.1007/s11060-016-2116-y

[17] Hegi, M.E., et al. (2005) MGMT Gene Silencing and Benefit from Temozolomide in Glioblastoma. The New England Journal of Medicine, 352, 997-1003. http://dx.doi.org/10.1056/NEJMoa043331

[18] Silber, J.R., Bobola, M.S., Blank, A. and Chamberlain, M.C. (2012) O ${ }^{6}$-Methylguanine-DNA Methyltransferase in Glioma Therapy: Promise and Problems. Biochimica et Biophysica Acta (BBA)—Reviews on Cancer, 1826, 71-82. http://dx.doi.org/10.1016/j.bbcan.2011.12.004

[19] D’Alessandris, Q.G., Montano, N., Cenci, T., et al. (2013) Targeted Therapy with Bevacizumab and Erlotinib Tailored to the Molecular Profile of Patients with Recurrent Glioblastoma. Preliminary Experience. Acta Neurochirurgica (Wien), 155, 33-40. http://dx.doi.org/10.1007/s00701-012-1536-5

[20] Hegi, M.E., Diserens, A.C., Godard, S., et al. (2004) Clinical Trial Substantiates the Predictive Value of O-6-Methylguanine-DNA Methyltransferase Promoter Methylation in Glioblastoma Patients Treated with Temozolomide. Clincal Cancer Research, 10, 1871-1874. http://dx.doi.org/10.1158/1078-0432.CCR-03-0384

[21] Gutenberg, A., Bock, H.C., Bruck, W., et al. (2013) MGMT Promoter Methylation Status and Prognosis of Patients with Primary or Recurrent Glioblastoma Treated with Carmustine Wafers. British Journal of Neurosurgery, 27, 772778. http://dx.doi.org/10.3109/02688697.2013.791664

[22] Mellai, M., Caldera, V., Annovazzi, L., et al. (2009) MGMT Promoter Hypermethylation in a Series of 104 Glioblastomas. Cancer Genomics Proteomics, 6, 219-227.

[23] Minniti, G., Salvati, M., Arcella, A., et al. (2011) Correlation between $\mathrm{O}^{6}$-Methylguanine-DNA Methyltransferase and Survival in Elderly Patients with Glioblastoma Treated with Radiotherapy plus Concomitant and Adjuvant Temozolomide. Journal of Neuro-Oncology, 102, 311-316. http://dx.doi.org/10.1007/s11060-010-0324-4

[24] Hegi, M.E. and Stupp, R. (2015) Withholding Temozolomide in Glioblastoma Patients with Unmethylated MGMT Promoter-Still a Dilemma? Neuro-Oncology, 17, 1425-1427. http://dx.doi.org/10.1093/neuonc/nov198

[25] Parney, I.F., Chang, L.J., Farr-Jones, M.A., Hao, C.H., Smylie, M. and Petruk, K.C. (2006) Technical Hurdles in a Pilot Clinical Trial of Combined B7-2 and GM-CSF Immunogene Therapy for Glioblastomas and Melanomas. Journal of Neuro-Oncology, 78, 71-80. http://dx.doi.org/10.1007/s11060-005-9058-0

[26] Shah, A., Bregy, A., Heros, D.O., Komotar, R.J. and Goldberg, J. (2013) Dendritic Cell Vaccine for Recurrent HighGrade Gliomas in Pediatric and Adult Subjects: Clinical Trial Protocol. Neurosurgery, 73, 863-867. http://dx.doi.org/10.1227/NEU.0000000000000107

[27] Ardon, H., Van Gool, S.W., Verschuere, T., et al. (2012) Integration of Autologous Dendritic Cell-Based Immunotherapy in the Standard of Care Treatment for Patients with Newly Diagnosed Glioblastoma: Results of the HGG-2006 Phase I/II Trial. Cancer Immunology, Immunotherapy, 61, 2033-2044. http://dx.doi.org/10.1007/s00262-012-1261-1

[28] Ceschin, R., Kurland, B.F., Abberbock, S.R., Ellingson, B.M., Okada, H., et al. (2015) Parametric Response Mapping of Apparent Diffusion Coefficient as an Imaging Biomarker to Distinguish Pseudoprogression from True Tumor Progression in Peptide-Based Vaccine Therapy for Pediatric Diffuse Intrinsic Pontine Glioma. American Journal of Neuroradiology, 36, 2170-2176. http://dx.doi.org/10.3174/ajnr.A4428

[29] Kebir, S., Fimmers, R., Galldiks, N., Schäfer, N., Mack, F., et al. (2015) Late Pseudoprogression in Glioblastoma: Diagnostic Value of Dynamic O-(2-[ ${ }^{18}$ F]fluoroethyl)-L-Tyrosine PET. Clinical Cancer Research, 22, 2190-2196.

[30] Thomas, A.A., Arevalo-Perez, J., Kaley, T., Lyo, J., Peck, K.K., et al. (2015) Dynamic Contrast Enhanced T1 MRI Perfusion Differentiates Pseudoprogression from Recurrent Glioblastoma. Journal of Neuro-Oncology, 125, $183-190$. http://dx.doi.org/10.1007/s11060-015-1893-z

[31] Gahramanov, S., Varallyay, C., Tyson, R.M., Lacy, C., Fu, R., et al. (2014) Diagnosis of Pseudoprogression Using MRI Perfusion in Patients with Glioblastoma Multiforme May Predict Improved Survival. CNS Oncology, 3, 389-400. http://dx.doi.org/10.2217/cns.14.42

[32] Nasseri, M., Gahramanov, S., Netto, J.P., Fu, R., Muldoon, L.L., et al. (2014) Evaluation of Pseudoprogression in Patients with Glioblastoma Multiforme Using Dynamic Magnetic Resonance Imaging with Ferumoxytol Calls RANO Criteria into Question. Neuro-Oncology, 16, 1146-1154. http://dx.doi.org/10.1093/neuonc/not328

[33] Macdonald, D.R., Cascino, T.L., Schold Jr., S.C. and Cairncross, J.G. (1990) Response Criteria for Phase II Studies of Supratentorial Malignant Glioma. Journal of Clinical Oncology, 8, 1277-1280.

[34] Vogelbaum, M.A., Jost, S., Aghi, M.K., Heimberger, A.B., et al. (2012) Application of Novel Response/Progression Measures for Surgically Delivered Therapies for Gliomas: Response Assessment in Neuro-Oncology (RANO) Working Group. Neurosurgery, 70, 234-243; discussion 243-244.

[35] Lazovic, J., Jensen, M.C., Ferkassian, E., et al. (2008) Imaging Immune Response in Vivo: Cytolytic Action of Genetically Altered T Cells Directed to Glioblastoma Multiforme. Clinical Cancer Research, 14, 3832-3839. 
http://dx.doi.org/10.1158/1078-0432.CCR-07-5067

[36] Cohen, J.V., Alomari, A.K., Vortmeyer, A.O., Jilaveanu, L.B., Goldberg, S.B., et al. (2016) Melanoma Brain Metastasis Pseudoprogression after Pembrolizumab Treatment. Cancer Immunology Research, 4, 179-182. http://dx.doi.org/10.1158/2326-6066.CIR-15-0160

[37] Fabi, A., Russillo, M., Metro, G., Vidiri, A., Di Giovanni, S. and Cognetti, F. (2009) Pseudoprogression and MGMT Status in Glioblastoma Patients: Implications in Clinical Practice. Anticancer Research, 29, 2607-2610.

[38] Hygino da Cruz, L.C., Rodriguez, I., Domingues, R.C., Gasparetto, E.L. and Sorensen, A.G. (2011) Pseudoprogression and Pseudoresponse: Imaging Challenges in the Assessment of Posttreatment Glioma. American Journal of Neuroradiology, 32, 1978-1985. http://dx.doi.org/10.3174/ajnr.A2397

[39] Brandes, A.A., Fransceschi, E., Tosoni, A., et al. (2008) MGMT Promoter Methylation Status Can Predict the Incidence and Outcome of Pseudoprogression after Concomitant Radiochemotherapy in Newly Diagnosed Glioblastoma Patients. Journal of Clinical Oncology, 26, 2192-2197. http://dx.doi.org/10.1200/JCO.2007.14.8163

[40] Reardon, D.A., Nabors, L.B., Mason, W.P., et al. (2015) Phase I/Randomized Phase II Study of Afatinib, an Irreversible ErbB Family Blocker, with or without Protracted Temozolomide in Adults with Recurrent Glioblastoma. NeuroOncology, 17, 430-439.

[41] Chauffert, B., Feuvret, L., Bonnetain, F., et al. (2014) Randomized Phase II Trial of Irinotecan and Bevacizumab as Neo-Adjuvant and Adjuvant to Temozolomide-Based Chemoradiation Compared with Temozolomide-Chemoradiation for Unresectable Glioblastoma: Final Results of the TEMAVIR Study from ANOCEF. Annals of Oncology, 25, 14421447. http://dx.doi.org/10.1093/annonc/mdu148

[42] Han, S.J., Rolston, J.D., Molinaro, A.M., et al. (2014) Phase II Trial of 7 Days on/7 Days off Temozolomide for Recurrent High-Grade Glioma. Neuro-Oncology, 16, 1255-1262. http://dx.doi.org/10.1093/neuonc/nou044

[43] Trippa, L., Wen, P.Y., Parmigiani, G., Berry, D.A. and Alexander, B.M. (2015) Combining Progression-Free Survival and Overall Survival as a Novel Composite Endpoint for Glioblastoma Trials. Neuro-Oncology, 17, 1106-1113. http://dx.doi.org/10.1093/neuonc/nou345

[44] Karsy, M., Neil, J.A., Guan, J., et al. (2015) A Practical Review of Prognostic Correlations of Molecular Biomarkers in Glioblastoma. Neurosurgical Focus, 38, E4. http://dx.doi.org/10.3171/2015.1.FOCUS14755

[45] Chen, J.R., Yao, Y., Xu, H.Z. and Qin, Z.-Y. (2016) Isocitrate Dehydrogenase (IDH)1/2 Mutations as Prognostic Markers in Patients with Glioblastomas. Medicine (Baltimore), 95, e2583. http://dx.doi.org/10.1097/MD.0000000000002583

[46] Montgomery, R.M., Queiroz, L.S. and Rogerio, F. (2015) EGFR, p53, IDH-1, and MDM2 Immunohistochemical Analysis in Glioblastoma: Therapeutic and Prognostic Correlation. Arquivos de Neuro-Psiquiatria, 73, 561-568. http://dx.doi.org/10.1590/0004-282X20150059

[47] Amelot, A., De Cremoux, P., Quillien, V., et al. (2015) IDH-Mutation Is a Weak Predictor of Long-Term Survival in Glioblastoma Patients. PLoS ONE, 10, e0130596. http://dx.doi.org/10.1371/journal.pone.0130596

[48] Labussiere, M., Boisselier, B., Mokhtari, K., et al. (2014) Combined Analysis of TERT, EGFR, and IDH Status Defines Distinct Prognostic Glioblastoma Classes. Neurology, 83, 1200-1206. http://dx.doi.org/10.1212/WNL.0000000000000814

[49] Nencha, U., Rahimian, A., Giry, M., et al. (2016) TERT Promoter Mutations and rs2853669 Polymorphism: Prognostic Impact and Interactions with Common Alterations in Glioblastoma. Journal of Neuro-Oncology, 126, 441-446. http://dx.doi.org/10.1007/s11060-015-1999-3

[50] Leonard, B., Starrett, G.J., Maurer, M.J., et al. (2016) APOBEC3G Expression Correlates with T Cell Infiltration and Improved Clinical Outcomes in High-Grade Serous Ovarian Carcinoma. Clinical Cancer Research. (Epub Ahead of Print) http://dx.doi.org/10.1158/1078-0432.CCR-15-2910

[51] Cescon, D.W., Haibe-Kains, B. and Mak, T.W. (2015) APOBEC3B Expression in Breast Cancer Reflects Cellular Proliferation, while a Deletion Polymorphism Is Associated with Immune Activation. Proceedings of the National Academy of Sciences of the United States of America, 112, 2841-2846. http://dx.doi.org/10.1073/pnas.1424869112

[52] Hamakawa, T., Kukita, Y., Kurokawa, Y., et al. (2015) Monitoring Gastric Cancer Progression with Circulating Tumour DNA. British Journal of Cancer, 112, 352-356. http://dx.doi.org/10.1038/bjc.2014.609

[53] Schwarzenbach, H., Alix-Panabieres, C., Muller, I., et al. (2009) Cell-Free Tumor DNA in Blood Plasma as a Marker for Circulating Tumor Cells in Prostate Cancer. Clinical Cancer Research, 15, 1032-1038. http://dx.doi.org/10.1158/1078-0432.CCR-08-1910

[54] Schwarzenbach, H., Muller, V., Milde-Langosch, K., Steinbach, B. and Pantel, K. (2011) Evaluation of Cell-Free Tumor DNA and RNA in Patients with Breast Cancer and Benign Breast Disease. Molecular BioSystems, 7, 2848-2854. http://dx.doi.org/10.1039/c1mb05197k 
[55] Luo, H., Li, H., Wu, H., et al. (2016) Noninvasive Diagnosis and Monitoring of Mutations by Deep Sequencing of Circulating Tumor DNA in Esophageal Squamous Cell Carcinoma. Biochemical and Biophysical Research Communications, 471, 596-602. http://dx.doi.org/10.1016/j.bbrc.2016.02.011

[56] Xu, S., Lou, F., Wu, Y., et al. (2016) Circulating Tumor DNA Identified by Targeted Sequencing in Advanced-Stage Non-Small Cell Lung Cancer Patients. Cancer Letters, 370, 324-331. http://dx.doi.org/10.1016/j.canlet.2015.11.005

[57] Dawson, S.J., Tsui, D.W., Murtaza, M., et al. (2013) Analysis of Circulating Tumor DNA to Monitor Metastatic Breast Cancer. The New England Journal of Medicine, 368, 1199-1209. http://dx.doi.org/10.1056/NEJMoa1213261

[58] Lipson, E.J., Velculescu, V.E., Pritchard, T.S., et al. (2014) Circulating Tumor DNA Analysis as a Real-Time Method for Monitoring Tumor Burden in Melanoma Patients Undergoing Treatment with Immune Checkpoint Blockade. Journal for ImmunoTherapy of Cancer, 2, 42. http://dx.doi.org/10.1186/s40425-014-0042-0

[59] Tie, J., Kinde, I., Wang, Y., et al. (2015) Circulating Tumor DNA as an Early Marker of Therapeutic Response in Patients with Metastatic Colorectal Cancer. Annals of Oncology, 26, 1715-1722. http://dx.doi.org/10.1093/annonc/mdv177

[60] Ocana, A., Diez-Gonzalez, L., Garcia-Olmo, D.C., et al. (2016) Circulating DNA and Survival in Solid Tumors. Cancer Epidemiology, Biomarkers \& Prevention, 25, 399-406. http://dx.doi.org/10.1158/1055-9965.EPI-15-0893

[61] Bidard, F.C., Madic, J., Mariani, P., et al. (2014) Detection Rate and Prognostic Value of Circulating Tumor Cells and Circulating Tumor DNA in Metastatic Uveal Melanoma. International Journal of Cancer, 134, 1207-1213. http://dx.doi.org/10.1002/ijc.28436

[62] Madic, J., Kiialainen, A., Bidard, F.C., et al. (2015) Circulating Tumor DNA and Circulating Tumor Cells in Metastatic Triple Negative Breast Cancer Patients. International Journal of Cancer, 136, 2158-2165. http://dx.doi.org/10.1002/ijc.29265

[63] Westphal, M. and Lamszus, K. (2015) Circulating Biomarkers for Gliomas. Nature Reviews Neurology, 11, $556-566$. http://dx.doi.org/10.1038/nrneurol.2015.171

[64] Herman, A., Gruden, K., Blejec, A., et al. (2015) Analysis of Glioblastoma Patients’ Plasma Revealed the Presence of MicroRNAs with a Prognostic Impact on Survival and Those of Viral Origin. PLoS ONE, 10, e0125791. http://dx.doi.org/10.1371/journal.pone.0125791

[65] Yan, J., Kong, L.Y., Hu, J., et al. (2015) FGL2 as a Multimodality Regulator of Tumor-Mediated Immune Suppression and Therapeutic Target in Gliomas. JNCI Journal of the National Cancer Institute, 107, djv137. http://dx.doi.org/10.1093/jnci/djv137

[66] Erdbruegger, U., Dhaygude, A., Haubiz, M. and Woywodt, A. (2010) Circulating Endothelial Cells: Markers and Mediators of Vascular Damage. Current Stem Cell Research \& Therapy, 5, 294-302. http://dx.doi.org/10.2174/157488810793351721

[67] Batchelor, T.T., Sorensen, A.G., di Tomaso, E., et al. (2007) AZD2171, a Pan-VEGF Receptor Tyrosine Kinase Inhibitor, Normalizes Tumor Vasculature and Alleviates Edema in Glioblastoma Patients. Cancer Cell, 11, 83-95. http://dx.doi.org/10.1016/j.ccr.2006.11.021

[68] Yong, W.H., Shabihkhani, M., Telesca, D., et al. (2015) Ribosomal Proteins RPS11 and RPS20, Two Stress-Response Markers of Glioblastoma Stem Cells, Are Novel Predictors of Poor Prognosis in Glioblastoma Patients. PLoS ONE, 10, e0141334. http://dx.doi.org/10.1371/journal.pone.0141334

[69] Sayour, E.J., McLendon, P., McLendon, R., et al. (2015) Increased Proportion of FoxP F $_{3}$ Regulatory T Cells in Tumor Infiltrating Lymphocytes Is Associated with Tumor Recurrence and Reduced Survival in Patients in GBM. Cancer Immunology, Immunotherapy, 64, 419-427. http://dx.doi.org/10.1007/s00262-014-1651-7

[70] Thomas, A.A., Fisher, J.L., Rahme, G.J., et al. (2015) Regulatory T Cells Are Not a Strong Predictor of Survival for Patients with GBM. Neuro-Oncology, 17, 801-809. http://dx.doi.org/10.1093/neuonc/nou363

[71] Gray, E.S., Rizos, H., Reid, A.L., et al. (2015) Circulating Tumor DNA to Monitor Treatment Response and Detect Acquired Resistance in Patients with Metastatic Melanoma. Oncotarget, 6, 42008-42018.

[72] Warton, K., Mahon, K.L. and Samimi, G. (2016) Methylated Circulating Tumor DNA in Blood: Power in Cancer Prognosis and Response. Endocrine-Related Cancer, 23, R157-R171. http://dx.doi.org/10.1530/ERC-15-0369

[73] Shah, K., Boghozian, R.A., Kartsonaki, C., et al. (2016) YH2AX Expression in Cytological Specimens as a Biomarker of Response to Radiotherapy in Solid Malignancies. Diagnostic Cytopathology, 44, 141-146. http://dx.doi.org/10.1002/dc.23396

[74] Hooper, C.E., Lyburn, I.D., Searle, J., et al. (2015) The South West Area Mesothelioma and Pemetrexed Trial: A Multicenter Prospective Observational Study Evaluating Novel Markers of Chemotherapy Response and Prognostication. British Journal of Cancer, 112, 1175-1182. http://dx.doi.org/10.1038/bjc.2015.62

[75] Mitchell, P.J., Welton, J., Staffurth, J., et al. (2009) Can Urinary Exosomes Act as Treatment Response Markers in Prostate Cancer? Journal of Translational Medicine, 7, 4. http://dx.doi.org/10.1186/1479-5876-7-4 
[76] Harder, J., Kummer, O., Olschewski, M., Otto, F., Blum, H.E. and Opitz, O. (2007) Prognostic Relevance of Carbohydrate Antigen 19-9 Levels in Patients with Advanced Biliary Tract Cancer. Cancer Epidemiology, Biomarkers \& Prevention, 16, 2097-2100. http://dx.doi.org/10.1158/1055-9965.EPI-07-0155

[77] Everson, R.G., Jin, R.M., Wang, X., et al. (2014) Cytokine Responsiveness of CD8 ${ }^{+}$T Cells Is a Reproducible Biomarker for the Clinical Efficacy of Dendritic Cell Vaccination in Glioblastoma Patients. Journal for ImmunoTherapy of Cancer, 2, 10. http://dx.doi.org/10.1186/2051-1426-2-10

[78] Muller, L., Muller-Haegele, S., Mitsuhashi, M., et al. (2015) Exosomes Isolated from Plasma of Glioma Patients Enrolled in a Vaccination Trial Reflect Antitumor Immune Activity and Might Predict Survival. OncoImmunology, 4, e1008347. http://dx.doi.org/10.1080/2162402X.2015.1008347

[79] Chen, H.Y., Li, X.Y., Li, W.B. and Zheng, H.Y. (2015) MiR-130a Can Predict Response to Temozolomide in Patients with Glioblastoma Multiforme, Independently of O6-Methylguanine-DNA Methyltransferance. Journal of Translational Medicine, 13, 69. http://dx.doi.org/10.1186/s12967-015-0435-y

[80] Comimelli, M., Grisanti, S., Mazzoleni, S., et al. (2015) EGFR Amplified and Overexpressing Glioblastomas and Associations with Better Response to Adjuvant Metronomic Temozolomide. JNCI Journal of the National Cancer Institute, 107, djv041.

[81] Lamano, J.B., Ampie, L., Choy, W., et al. (2016) Immunomonitoring in Glioma Immunotherapy: Current Status and Future Perspectives. Journal of Neuro-Oncology, 127, 1-13. http://dx.doi.org/10.1007/s11060-015-2018-4

[82] Mazmanian, S.K., Round, J.L. and Kasper, D.L. (2008) A Microbial Symbiosis Factor Prevents Intestinal Inflammatory Disease. Nature, 453, 620-625. http://dx.doi.org/10.1038/nature07008

[83] Cash, H.L. (2006) Symbiotic Bacteria Direct Expression of an Intestinal Bactericidal Lectin. Science, 313, $1126-1130$. http://dx.doi.org/10.1126/science.1127119

[84] Berman, D., Parker, S.M., Siegel, J., Chasalow, S.D., Weber, J., et al. (2010) Blockade of Cytotoxic T-Lymphocyte Antigen-4 by Ipilimumab Results in Dysregulation of Gastrointestinal Immunity in Patients with Advanced Melanoma. Cancer Immunity, 10, 11.

[85] Vetizou, M., Pitt, J.M., Daillere, R., et al. (2015) Anticancer Immunotherapy by CTLA-4 Blockade Relies on the Gut Microbiota. Science, 350, 1079-1084. http://dx.doi.org/10.1126/science.aad1329

[86] Sivan, A., Corrales, L., Hubert, N., Williams, J.B., Aquino-Michaels, K., et al. (2015) Commensal Bifidobacterium Promotes Antitumor Immunity and Facilitates Anti-PD-L1 Efficacy. Science, 350, 1084-1089. http://dx.doi.org/10.1126/science.aac4255

[87] Linhares, P., Carvalho, B., Figueiredo, R., Reis, R.M. and Vaz, R. (2013) Early Pseudoprogression Following Chemoradiotherapy in Glioblastoma Patients: The Value of RANO Evaluation. Journal of Oncology, 2013, Article ID: 690585. http://dx.doi.org/10.1155/2013/690585

[88] Bulik, M., Kazda, T., Slampa, P. and Jancalek, R. (2015) The Diagnostic Ability of Follow-Up Imaging Biomarkers after Treatment of Glioblastoma in the Temozolomide Era: Implications from Proton MR Spectoscopy and Apparent Diffusion Coefficient Mapping. BioMed Research International, 2015, Article ID: 641023. http://dx.doi.org/10.1155/2015/641023

[89] Kelm, Z.S., Korfiatis, P.D., Lingineni, R.K., et al. (2015) Variability and Accuracy of Different Software Packages for Dynamic Susceptibility Contrast Magnetic Resonance Imaging for Distinguishing Glioblastoma Progression from Pseudoprogression. Journal of Medical Imaging, 2, 026001. http://dx.doi.org/10.1117/1.JMI.2.2.026001

[90] Galldiks, N., Dunkl, V., Stoffels, G., et al. (2015) Diagnosis of Pseudoprogression in Patients with Glioblastoma Using O-(2-[ $\left[{ }^{18} \mathrm{~F}\right]$ fluoroethyl)-L-tyrosine PET. European Journal of Nuclear Medicine and Molecular Imaging, 42, 685-695. http://dx.doi.org/10.1007/s00259-014-2959-4

[91] Cha, J., Kim, S.T., Kim, H.J., et al. (2014) Differentiation of Tumor Progression from Pseudoprogression in Patients with Posttreatment Glioblastoma Using Multiparametric Histogram Analysis. American Journal of Neuroradiology, 35 , 1309-1317. http://dx.doi.org/10.3174/ajnr.A3876

[92] Park, J.E., Kim, H.S., Goh, M.J., Kim, S.J. and Kim, J.H. (2015) Pseudoprogression in Patients with Glioblastoma: Assessment by Using Volume-Weighted Voxel-Based Multiparametric Clustering of MR Imaging Data in an Independent Test Set. Radiology, 275, 792-802. http://dx.doi.org/10.1148/radiol.14141414

[93] Suh, C.H., Kim, H.S., Choi, Y.J., Kim, N. and Kim, S.J. (2013) Prediction of Pseudoprogression in Patients with Glioblastomas Using the Initial and Final Area under the Curves Ratio Derived from Dynamic Contrast-Enhanced T1Weighted Perfusion MR Imaging. American Journal of Neuroradiology, 34, 2278-2286. http://dx.doi.org/10.3174/ajnr.A3634

[94] Song, Y.S., Choi, S.H., Park, C.K., et al. (2013) True Progression versus Pseudoprogression in the Treatment of Glioblastomas: A Comparison Study of Normalized Cerebral Blood Volume and Apparent Diffusion Coefficient by Histogram Analysis. Korean Journal of Radiology, 14, 662-672. http://dx.doi.org/10.3348/kjr.2013.14.4.662 
[95] Gahramanov, S., Muldoon, L.L., Varallyay, C.G., et al. (2013) Pseudoprogression of Glioblastoma after Chemo- and Radiation Therapy: Diagnosis by Using Dynamic Susceptibility-Weighted Contrast-Enhanced Perfusion MR Imaging with Ferumoxytol versus Gadoteridol and Correlation with Survival. Radiology, 266, 842-852. http://dx.doi.org/10.1148/radiol.12111472

[96] Chu, H.H., Choi, S.H., Ryoo, I., et al. (2013) Differentiation of True Progression from Pseudoprogression in Glioblastoma Treated with Radiation Therapy and Concomitant Temozolomide: Comparison Study of Standard and High- $b$ Value Diffusion-Weighted Imaging. Radiology, 269, 831-840. http://dx.doi.org/10.1148/radiol.13122024

[97] Laymon, C.M., Oborski, M.J., Lee, V.K., et al. (2012) Combined Imaging Biomarkers for Therapy Evaluation in Glioblastoma Multiforme: Correlating Sodium MRI and F-18 FLT PET on a Voxel-Wise Basis. Magnetic Resonance Imaging, 30, 1268-1278. http://dx.doi.org/10.1016/j.mri.2012.05.011

[98] Cairncross, J.G., Wang, M., Jenkins, R.B., et al. (2014) Benefit from Procarbazine, Lomustine, and Vincristine in Oligodendroglial Tumors Is Associated with Mutation of IDH. Journal of Clinical Oncology, 32, 783-790. http://dx.doi.org/10.1200/JCO.2013.49.3726

[99] Balmanoukian, A., Ye, X., Herman, J., Laheru, D. and Grossman, S.A. (2012) The Association between TreatmentRelated Lymphopenia and Survival in Newly Diagnosed Patients with Resected Adenocarcinoma of the Pancreas. Cancer Investigation, 30, 571-576. http://dx.doi.org/10.3109/07357907.2012.700987

[100] Grossman, S.A., Ellsworth, S., Campian, J., et al. (2015) Survival in Patients with Severe Lymphopenia Following Treatment with Radiation and Chemotherapy for Newly Diagnosed Solid Tumors. Journal of the National Comprehensive Cancer Network, 13, 1225-1231.

[101] Ellsworth, S., Balmanoukian, A., Kos, F., et al. (2014) Sustained CD4 ${ }^{+}$T Cell-Driven Lymphopenia without a Compensatory IL-7/IL-15 Response among High-Grade Glioma Patients Treated with Radiation and Temozolomide. OncoImmunology, 3, e27357. http://dx.doi.org/10.4161/onci.27357

[102] Wen, P.Y., Cloughesy, T.F., Ellingson, B.M., et al. (2014) Report of the Jumpstarting Brain Tumor Drug Development Coalition and FDA Clinical Trials Neuroimaging Endpoint Workshop. Neuro-Oncology, 16, 36-47. http://dx.doi.org/10.1093/neuonc/nou226

[103] Floeth, F.W., Wittsack, H.J., Engelbrecht, V. and Weber, F. (2002) Comparative Follow-Up of Enhancement Phenomena with MRI and Proton MR Spectroscopic Imaging after Intralesional Immunotherapy in Glioblastoma-Report of Two Exceptional Cases. Zentralbl Neurochir, 63, 23-28. http://dx.doi.org/10.1055/s-2002-31579

[104] Yang, I., Huh, N.G., Smith, Z.A., et al. (2010) Distinguishing Glioma Recurrence from Treatment Effect after Radiochemotherapy and Immunotherapy. Neurosurgery Clinics of North America, 21, 181-186. http://dx.doi.org/10.1016/j.nec.2009.08.003

[105] Reardon, D.A., Akabani, G., Coleman, R.E., et al. (2002) Phase II Trial of murine ${ }^{131}$ I-Labeled Antitenascin Monoclonal Antibody 81C6 Administered into Surgically Created Resection Cavities of Patients with Newly Diagnosed Malignant Gliomas. Journal of Clinical Oncology, 20, 1389-1397. http://dx.doi.org/10.1200/JCO.20.5.1389

[106] Tran, E., Ahmadzadeh, M., Lu, Y.C., et al. (2015) Immunogenicity of Somatic Mutations in Human Gastrointestinal Cancers. Science, 350, 1387-1390. http://dx.doi.org/10.1126/science.aad1253

[107] Chan, T.A., Wolchok, J.D. and Snyder, A. (2015) Genetic Basis for Clinical Response to CTLA-4 Blockade in Melanoma. The New England Journal of Medicine, 373, 1984. http://dx.doi.org/10.1056/NEJMc1508163

\section{Submit or recommend next manuscript to SCIRP and we will provide best service for you:}

Accepting pre-submission inquiries through Email, Facebook, LinkedIn, Twitter, etc.

A wide selection of journals (inclusive of 9 subjects, more than 200 journals)

Providing 24-hour high-quality service

User-friendly online submission system

Fair and swift peer-review system

Efficient typesetting and proofreading procedure

Display of the result of downloads and visits, as well as the number of cited articles

Maximum dissemination of your research work

Submit your manuscript at: http://papersubmission.scirp.org/ 